\title{
Há correlação entre o nível de atividade auto-referido e desempenho físico observado em mulheres idosas?
}

\author{
Is there a correlation between self-reported level of activity and observed physical \\ performance in elderly women?
}

Renata Marinho Pereira ${ }^{1}$, Silvio Lopes Alabarse, ${ }^{2}$ Renata Cereda Cordeiro ${ }^{3}$

Estudo desenvolvido no Depto. de Medicina Preventiva da EPM/Unifesp - Escola Paulista de Medicina da Universidade Federal de São Paulo, São Paulo, SP, Brasil

1 Fisioterapeuta especializada em Reabilitação Gerontológica

2 Educador físico Ms.

3 Fisioterapeuta Ms. Coordenadora do Setor de Reabilitação Gerontológica do Lar Escola São Francisco, vinculado à EPM/Unifesp

\section{ENDEREÇO PARA}

CORRESPONDÊNCIA

Renata M. Pereira

R. Paula Ney 705 ap.1001 Aldeota

60140-200 Fortaleza CE e-mail:

renatafisio2005@yahoo.com.br

APRESENTAÇÃO

nov. 2007

ACEITO PARA PUBLICAÇÃO ago. 2008
Resumo: Este estudo visou verificar se havia associação entre o nível de atividade físico-funcional auto-referido e o desempenho físico de idosas em centro de

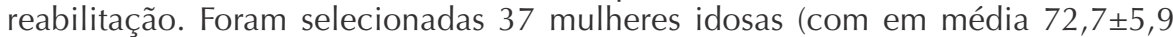
anos) pelas fichas de admissão em serviço de atividade física de um centro de referência em reabilitação. Para a avaliação do nível de atividade física autoreferido empregou-se o Perfil de Atividade Humana (PAH); na observação direta de desempenho físico aplicaram-se os testes de caminhada em 6 minutos (TC6') e levantar da cadeira em 30 segundos (TSL30"). Foi encontrada correlação positiva fraca $(r=0,41)$ e estatisticamente significante $(p=0,01)$ entre os valores do PAH e o TC6'; e uma tendência de associação entre a classificação do nível de atividade e a variável categórica TC6' $(p=0,08)$. Também foi encontrada correlação positiva fraca $(r=0,36)$ e estatisticamente significante $(p=0,02)$ entre o PAH e o TSL30". Pode-se afirmar que o auto-relato de nível de atividade física das idosas estudadas correlaciona-se, porém fracamente, ao desempenho físico observado. Sugerem-se novas pesquisas com amostras representativas da população idosa em uma comunidade, não apenas de pacientes de centro de reabilitação, para averiguar possíveis tendências à subestimação ou superestimação do nível de atividade física em avaliações auto-referidas.

Descritores: Análise e desempenho de tarefas; Atividades cotidianas; Idoso; Mulheres

ABSTRACT: The aim of the study was to verify the degree of association between self-reported level of physical activity and observed physical performance of aged women. Thirty-seven women (mean aged $72.7 \pm 5.99$ years old) were selected by looking up admission records in a reference centre for physical rehabilitation. The Human Activity Profile (HAP) was used to measure activity level; and, for direct observation of physical performance, the six-minute walk test (6MWT) and the 30-second chair-stand test were applied. A statistically significant $(p=0.01)$, weak positive correlation $(r=0.41)$ was found between HAP scores and 6MWT values; and an association trend was noticed between the classification of activity level and the 6MWT variable $(p=0.08)$. Also, a significant $(p=0.02)$, weak positive correlation $(r=0.36)$ was found between HAP scores and results of the 30-second chair stand test. It may be said there is a correlation, though weak, between self-reported and observed levels of physical performance among the elderly here studied. Further research should address aged community dwellers - not only rehabilitation patients - in order to assess a possible under or overestimation of the physical activity level in self-reported evaluations.

KEY wORDs: Activities of daily living; Aged; Task performance and analysis; Women 


\section{INTRODUÇÃO}

O crescimento da população de idosos é um fenômeno mundial e está ocorrendo a um nível sem precedentes. Considerando a continuidade das tendências das taxas de fecundidade e longevidade da população brasileira, as estimativas para os próximos 20 anos indicam que a população idosa poderá exceder 30 milhões de pessoas, chegando a representar quase $13 \%$ da população ${ }^{1}$.

O envelhecimento é conceituado como um processo dinâmico e progressivo, em que ocorrem alterações morfológicas e bioquímicas no organismo, tornando-o mais suscetível às agressões intrínsecas e extrínsecas que culminam na morte ${ }^{2}$. As alterações estruturais e funcionais, embora variem individualmente, são encontradas em todos os idosos e próprias do processo de envelhecimento normal ${ }^{3}$. O envelhecimento biológico resulta em inevitável diminuição da capacidade fisiológica. O declínio dos principais sistemas do organismo (cardiovascular, metabólico, respiratório e neuromuscular) contribui para fraqueza, fadiga e movimentos mais lentos, tidos como os principais marcadores do envelhecimento ${ }^{4}$. A proporção de massa muscular no adulto jovem, que chega a $19 \%$, cai para $12 \%$ no idoso. A sarcopenia (perda de massa muscular ligada ao envelhecimento) é associada a níveis restritos de atividade, redução do metabolismo basal e da força muscular ${ }^{5}$. Esta atinge mais precocemente seu auge nas mulheres, em quem a perda de força também se inicia antes da dos homens. Apesar dessas diferenças, o decréscimo da força muscular, ao longo do envelhecimento, parece ser equivalente para ambos os sexos ${ }^{6}$.

A capacidade de pessoas idosas de realizar atividades aeróbicas, como andar e correr, é adversamente afetada pelo declínio do consumo máximo de oxigênio $\left(\mathrm{VO}_{2 \text { máx }}\right)$, de cerca de 0,5 a $1,0 \%$ ao ano. Em indivíduos saudáveis e sedentários, $\mathrm{o} \mathrm{VO}_{2 \text { máx }}$ diminui $1,3 \mathrm{l} / \mathrm{min}$ nas mulheres e 2,0 $\mathrm{l} / \mathrm{min}$ em homens dos 25 aos 85 anos $^{7}$. A regressão do $\mathrm{VO}_{2 \text { máx }}$ ao longo da vida corresponde à perda progressiva da capaci- dade de trabalho e de engajamento em uma diversidade de tarefas motoras. Muitos indivíduos sedentários entre 65 e 85 anos, período em que ocorrem perdas mais severas, podem atingir níveis críticos de consumo máximo de oxigênio, inviabilizando a autonomia motora. Em síntese, a diminuição do $\mathrm{VO}_{2 \text { máx }}$ no decorrer do envelhecimento advém da diminuição da freqüência cardíaca máxima, da massa muscular, da capacidade de redirecionamento do fluxo sangüíneo e da capacidade dos músculos de utilizar o oxigênio ${ }^{8}$. O consumo de oxigênio reflete-se na habilidade de idosos saudáveis em realizar atividades da vida diária, como transferências da cama para a cadeira, ir ao banheiro, vestirse, tomar banho, preparar refeições e caminhar $^{9}$. Assim, a manutenção da capacidade aeróbica é o maior contribuinte para a manutenção da independência funcional em idosos ${ }^{10}$.

Define-se capacidade funcional (CF) como "o grau de preservação da habilidade em executar, de forma autônoma e independente, as atividades básicas de vida diária (de autocuidado) e instrumentais de vida diária (mais complexas, auxiliares), dependentes de habilidades físicas e mentais"11. CF surge então como um novo conceito ou paradigma de saúde, marcado pelo fato de o declínio funcional constituir forte preditor de mortalidade entre idosos ${ }^{12,13}$. Considerando-se a relevância da avaliação da CF, fundamentada nos conceitos de funcionalidade e saúde introduzidos pela Organização Mundial de Saúde, têm sido criados instrumentos padronizados e psicometricamente rigorosos para descrever adequadamente a capacidade funcional de populações específicas de idosos (em hospitais, instituições de longa permanência, comunidade, ambulatórios) ${ }^{14}$. Um desses instrumentos é o Perfil de Atividade Humana $(\mathrm{PAH})^{14}$, de auto-relato das atividades físicas. O objetivo deste trabalho foi verificar em que medida o nível de atividade físico-funcional auto-referido no $\mathrm{PAH}$ se relaciona com o desempenho físico de idosas nos testes de caminhada de 6 minutos e de levantar da cadeira em 30 segundos.

\section{METODOLOGIA}

Este é um estudo do tipo descritivo, exploratório, de corte transversal. Para localização das participantes, foram levantadas as fichas de avaliação de pacientes encaminhados para o Setor de Atividade Física Adaptada do Centro de Reabilitação Lar Escola São Francisco, vinculado à Unifesp.

Foram incluídas no estudo mulheres com idade igual ou maior de 60 anos, admitidas no serviço no período de maio de 2006 a janeiro de 2007, somando um total de 37 idosas. A amostra foi pois não-probabilística, consecutiva, respeitando-se a ordem de chegada ao serviço. Os indivíduos do sexo masculino foram excluídos por sua ínfima ocorrência na amostra (três). Essa decisão tem por fundamento os achados de Bennell et al. ${ }^{15}$, de que mulheres com osteoartrite (OA) de joelhos tenderam a menores escores no Perfil de Atividade Humana $(\mathrm{PAH})$ que os homens (cujos escores entre aqueles com e sem a doença não diferiram significantemente, ao contrário das mulheres). Sabendo-se ser o PAH menos sensível em homens do que em mulheres e discriminativo em relação ao sexo ${ }^{16}$, isso implicaria controle dessa variável e o número exíguo de homens não permitiria conduzir análise estatística.

Todos os dados utilizados neste estudo foram extraídos da avaliação de triagem que separaria os participantes para ingresso nos seus respectivos grupos de atendimento (iniciante, intermediário e avançado), planejados de acordo com as habilidades físicas de cada grupo. Todas as pacientes encontravam-se pois em fase de "reabilitação preventiva". Foram utilizadas apenas informações coletadas em prontuários, todas obtidas previamente por avaliadores fisioterapeutas treinados para a aplicação dos testes de desempenho e dos questionários. Além dos dados destes últimos, foram coletados dados de identificação e clínicos de doenças autoreferidas: dor no peito, dor no corpo inteiro, dor na coluna, reumatismos, diabetes, hipertensão, dislipidemia, obesidade, quedas, fraqueza, problemas de visão e de audição. 


\section{Perfil de Atividade Humana (PAH)}

O PAH é um questionário utilizado na avaliação do nível funcional e de atividade física, tanto para indivíduos saudáveis, em qualquer faixa etária, quanto para aqueles com algum grau de disfunção ${ }^{14}$. É composto de 94 itens, de atividades rotineiras de variados níveis funcionais, ordenados de acordo com o custo energético: os de menor numeração demandam menor gasto energético, os de numeração mais alta, maior gasto energético. Para cada item, há três respostas possíveis: "ainda faço", "parei de fazer", ou "nunca fiz", sendo que esta última não é computada em qualquer escore ou classificação. A ampla variação dos escores, contemplando atividades das mais básicas às mais avançadas em termos de aptidão física, é uma característica que evita efeitos chão-e-teto ${ }^{17}$. Para pontuação, é obtido o escore máximo de atividade (EMA), representado pela atividade de maior consumo de oxigênio que o indivíduo ainda realiza ${ }^{18}$. Em seguida, calcula-se o escore ajustado de atividade (EAA), subtraindo-se do último item cuja resposta é "ainda faço" o número de atividades que ele parou de fazer, anteriores ao item que o indivíduo ainda $\mathrm{faz}^{14}$ (assim, o escore máximo seria 94, ou seja, atividade física máxima, e o mínimo, zero). O EMA é considerado uma medida do nível de atividade física, enquanto EAA, uma medida de funcionalidade física ${ }^{18}$. O EAA fornece uma estimativa mais precisa das atividades diárias, pois representa o nível médio de equivalente metabólico gasto em um dia típico. A classificação é obtida pelo número do EAA, sendo o sujeito considerado ativo $(E A A>74)$, moderadamente ativo $(53 \leq \mathrm{EAA} \leq 74)$ ou prejudicado $(\mathrm{EAA}<53)^{14}$.

\section{Teste da caminhada em 6 minutos}

A principal indicação do teste da caminhada em 6 minutos (TC6') é medir a resposta à intervenção medicamentosa em pacientes com doença cardíaca ou pulmonar moderada a severa, mas também tem sido utilizado como medida da CF, bem como prognóstico de morbidade e mortalidade $^{19}$. Esse protocolo analisa a via de transferência de energia predominantemente aeróbica, pois considera-se o sistema aeróbico como de longo prazo e baixa intensidade, em que o consumo de oxigênio aumenta exponencialmente durante os primeiros minutos do exercício para atingir um platô entre o terceiro e o quarto minutos em atividade física estável ${ }^{20}$.

Este teste foi aplicado em um corredor onde eram marcados 15 metros de comprimento, delimitados por dois cones, com placas fixas na parede a cada metro. Explicava-se às pacientes que o objetivo do teste era verificar o máximo que elas poderiam andar durante 6 minutos. Elas então eram instruídas a caminhar na área demarcada, no próprio passo, tentando andar o máximo possível ${ }^{19}$. A cada minuto o avaliador encorajava as participantes com frases como: "você está indo bem" ou "continue assim, bom trabaIho" 21 , somente observando-as, sem caminhar ao lado das avaliadas para não haver influência na realização do teste. O tempo era marcado com um cronômetro pelo avaliador que anotava o número de voltas e ao final calculava a distância caminhada.

Para encontrar o valor predito para cada paciente, foi utilizada a equação de referência para o TC6' para mulheres ${ }^{22}$ : distância predita $=(2,11 \mathrm{x}$ alturacm $)-(2,29 \times$ pesokg $)-(5,78 \times$ idade) $+667 \mathrm{~m}$. De acordo com Enright et al. ${ }^{21}$, foi considerada nota de corte o limite mais baixo de normalidade, que é de aproximadamente $75 \%$ do valor predito.

\section{Teste de levantar da cadeira em 30 segundos}

Esse teste é uma alternativa prática para medir indiretamente a força dos membros inferiores devido à correlação moderadamente alta com o teste de uma repetição máxima no leg press em homens $(0,78)$ e mulheres $(0,71)^{23}$. Avalia a potência e capacidade anaeróbica alática, que representa atividade de alta intensidade e curta duração (até 30 segundos), mas que exige esforço máximo e necessita de uma libe- ração quase imediata de energia. Assim, esse teste de campo prático é destinado a avaliar a capacidade de transferência dessa energia imediata ${ }^{24}$.

A paciente avaliada sentava-se com a coluna reta, os pés apoiados no chão e os braços cruzados contra o tórax em uma cadeira sem braços, que devia estar encostada em uma parede. Após o sinal do avaliador, a paciente devia levantar-se, ficando totalmente de pé, retornando em seguida à posição sentada e repetindo o movimento. Todas eram encorajadas a levantar-se completamente o maior número possível de vezes durante 30 segundos $^{23}$.

Jones et al. ${ }^{23}$ calcularam a média de repetições de acordo com a faixa etária e com o nível de atividade física dos pacientes. Estratificaram sua amostra da seguinte forma: de 60 a 69 anos, a média foi de $14 \pm 2,4$ repetições; de 70 a 79 anos foi de $12,9 \pm 3,0$; e de 80 a 89 anos, de $11,9 \pm 3,6$. Os pacientes mais ativos apresentaram média de $13,3 \pm 2,8$ e os menos ativos média

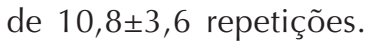

\section{Análise estatística}

As variáveis categóricas foram descritas em freqüências absolutas e relativas; as quantitativas de distribuição normal foram descritas por média e desvio padrão, enquanto as de distribuição assimétrica, como mediana e intervalo interquartil. O teste de Kolmogorov-Smirnov foi utilizado para verificar a de normalidade de variáveis quantitativas ${ }^{25}$. A análise de correlação linear (com IC=95\%) foi efetuada utilizando-se o coeficiente de correlação de Pearson ${ }^{26}$. Correlações abaixo de 0,3 foram consideradas inexistentes; entre 0,3 e 0,5, fracas; entre 0,5 e 0,7 , moderadas; e, acima de 0,7 , correlações fortes. Intervalos de confiança de $95 \%$ foram calculados para o coeficiente de correlação de Pearson segundo a transformação Z de Fisher $^{27}$.

Para testar associação entre duas variáveis categóricas em tabelas de contingência maiores que $2 \times 2$, utilizou-se o teste exato de FisherFreeman-Halton, uma generalização do teste exato de Fisher ${ }^{28}$. 
Todas as probabilidades de significância (valores de $p$ ) foram do tipo bilateral, considerando-se significantes $p<0,05$; valores de $p$ entre 0,05 e 0,10 foram interpretados como marginalmente significantes.

A análise estatística foi efetuada utilizando-se SAS versão 9.1.3 (Statistical Analysis System, Cary, NC, USA) e Minitab versão 15.1 (Minitab Inc, State College, PA, USA).

\section{RESULTADOS}

A amostra foi composta por 37 muIheres com idade média de $72,7 \pm 6,0$ anos, ocorrendo a maior concentração de idade na faixa de 70 a 79 (46\%), sendo a idade mínima de 62 e a máxima de 85 anos. A estatura média foi $151 \pm 6,1 \mathrm{~cm}$ e massa média, $65 \pm 11,6 \mathrm{~kg}$. A aferição da pressão arterial realizada em repouso apresentou valores médios de pressão arterial sistólica de 130 $\pm 13,6$ mmHg e diastólica de $79 \pm 10,3 \mathrm{mmHg}$, ou seja, $89,2 \%$ das mulheres eram normotensas durante a verificação. No entanto, segundo o relato das patologias auto-referidas (Tabela 1), 29 pacientes $(78,3 \%)$ eram hipertensas. O mesmo ocorreu para o relato de obesidade pois, no auto-relato, 70,3\% da amostra não se considerou obesa ou com dificuldade em diminuir o peso corporal (Tabela 1) mas, na avaliação realizada pelos examinadores, encontrou-se o índice médio de

Tabela 1 Distribuição das doenças auto-referidas na amostra

\begin{tabular}{lcc}
\hline Doenças auto-referidas & $\mathrm{n}$ & $\%{ }^{*}$ \\
\hline Dor no peito & 12 & 32,4 \\
Dor no corpo inteiro & 15 & 40,6 \\
Dor na coluna & 29 & 78,4 \\
Reumatismos & $21^{*}$ & 58,3 \\
Diabetes & $6^{*}$ & 16,7 \\
Hipertensão & 29 & 78,4 \\
Dislipidemia & $17^{*}$ & 47,2 \\
Obesidade & 11 & 29,7 \\
Quedas & $14 *$ & 38,9 \\
Fraqueza & 11 & 29,7 \\
Problemas de visão & 22 & 59,5 \\
Problemas de audição & 16 & 43,2 \\
\hline *n=36; percentual válido & &
\end{tabular}

* $\mathrm{n}=36$; percentual válido

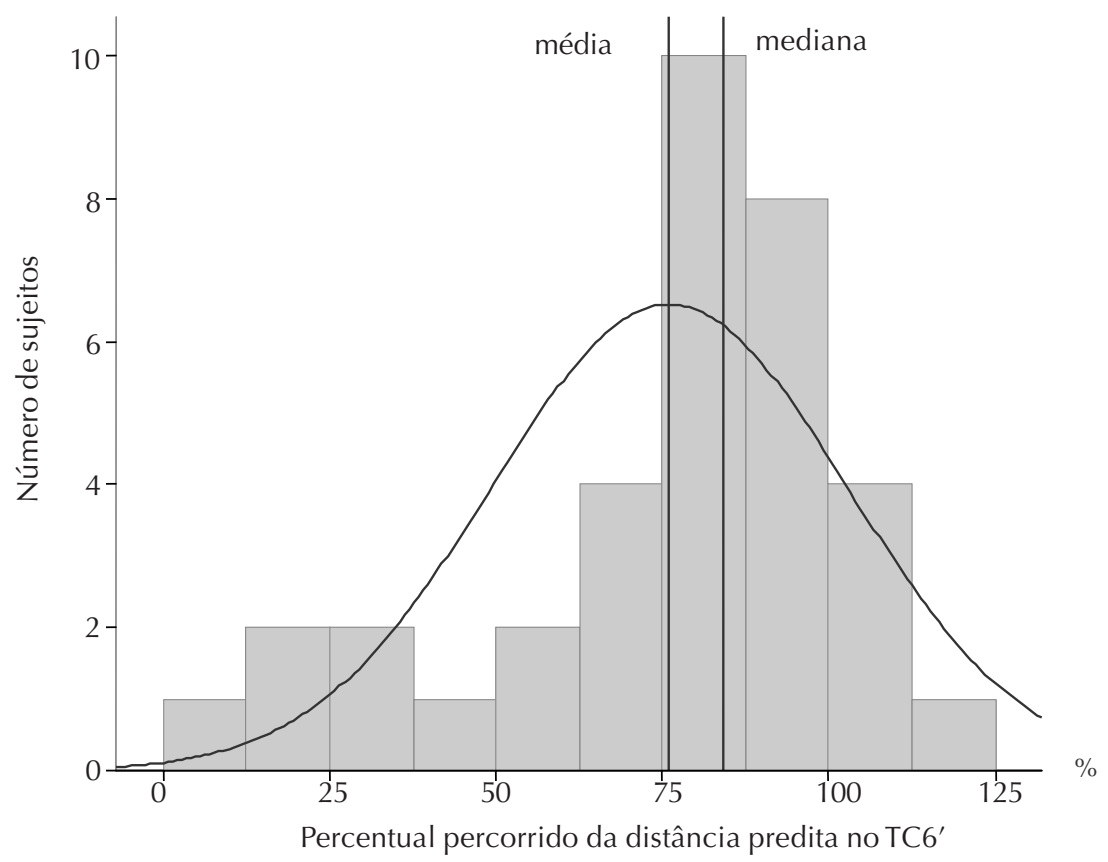

Gráfico 1 Distância percorrida pelas idosas (\% em relação à prevista) no teste da caminhada de 6 minutos (TC6') $n=35$

massa corpórea (IMC) de $28 \pm 4,7 \mathrm{~kg} / \mathrm{m}^{2}$, indicando $61,1 \%$ de idosas obesas $\left(\mathrm{IMC}>27 \mathrm{~kg} / \mathrm{m}^{2}\right)$.

De acordo com o PAH, 70,2\% das pacientes foram classificadas como moderadamente ativas, 21,7\% como prejudicadas e apenas $8,1 \%$ como ativas, sendo a média \pm desvio padrão encontrada de $60 \pm 13,1$ pontos. O menor escore atingido foi 23 e o máximo foi 88 .

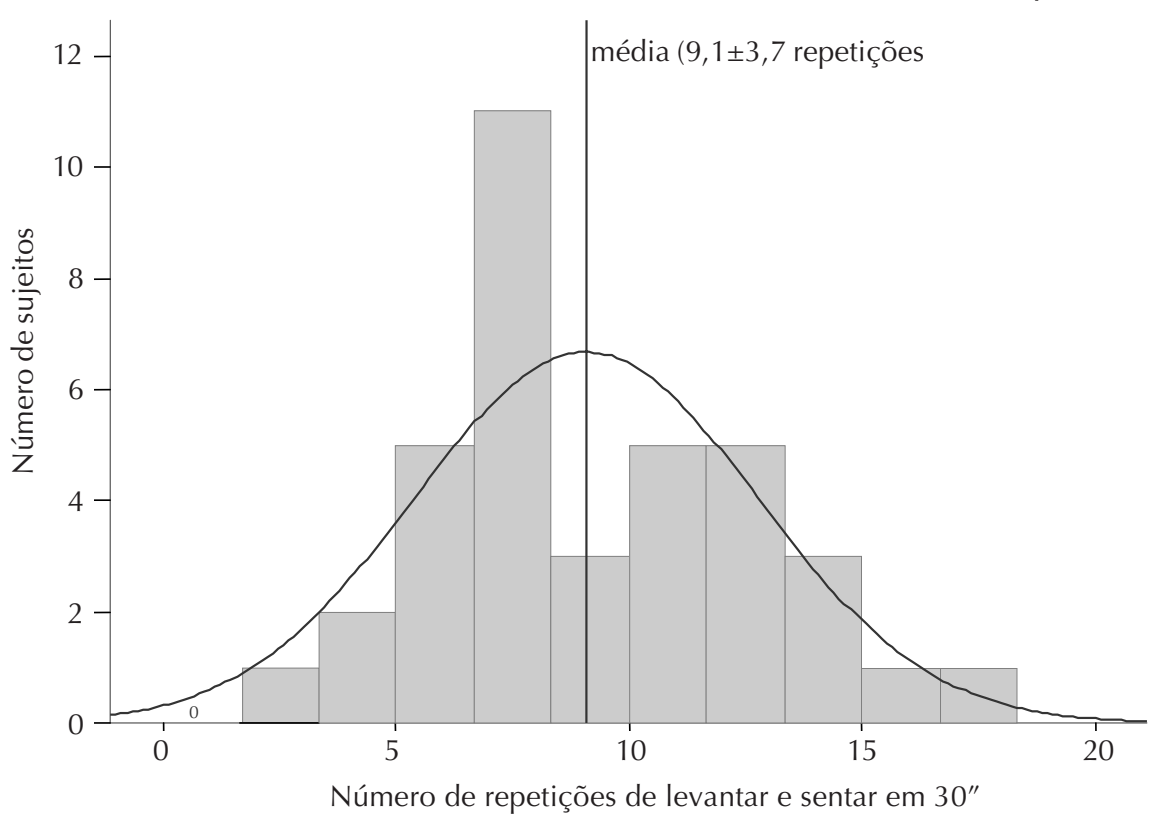

Gráfico 2 Número de repetições no teste de levantar da cadeira em 30 segundos (TSL30") $\mathrm{n}=37$

No TC6 ${ }^{\prime}$, considera-se que sujeitos percorrendo menos de $75 \%$ da distância prevista teriam pior condição física aeróbica. Neste estudo a mediana foi de $84 \pm 26,8 \%$ da distância percorrida em relação à prevista, com valor mínimo de 1,4 e máximo de $120,3 \%$. Dentre as participantes, 23 $(65,7 \%)$ e $12(34,3 \%)$ percorreram respectivamente distâncias acima e abaixo de $75 \%$ da distância prevista média $(9,1 \pm 3,7$ repetições . 
para cada uma (Gráfico 1). Duas pacientes não conseguiram finalizar o teste, com queixas de dispnéia e dor nos membros inferiores, reduzindo portanto a amostra para 35 sujeitos.

No teste de levantar da cadeira em 30 segundos (TSL30"), a amostra deste estudo apresentou média de $9 \pm 3,7$ repetições, com mínimo de uma e máximo de 18 repetições; 18 idosas $(48,6 \%)$ fizeram mais de 9 repetições e 19 (51,4\%) fizeram menos do que a média (Gráfico 2).
Ao verificar a relação entre os resultados do PAH e do TC6', encontrou-se uma correlação positiva fraca $(r=0,41)$ e estatisticamente significativa $(p=0,01)$ (Gráfico 3). Quanto ao desempenho no TC6' em relação à classificação funcional do $\mathrm{PAH}, 67 \%$ do grupo ativo, $76 \%$ do grupo moderadamente ativo e $28,5 \%$ do grupo prejudicado conseguiram caminhar $75 \%$ ou mais da distância prevista durante o teste. Observou-se também uma tendência de associação entre essa classificação e a variável TC6' (nota de corte em $75 \%, p=0,08)$.

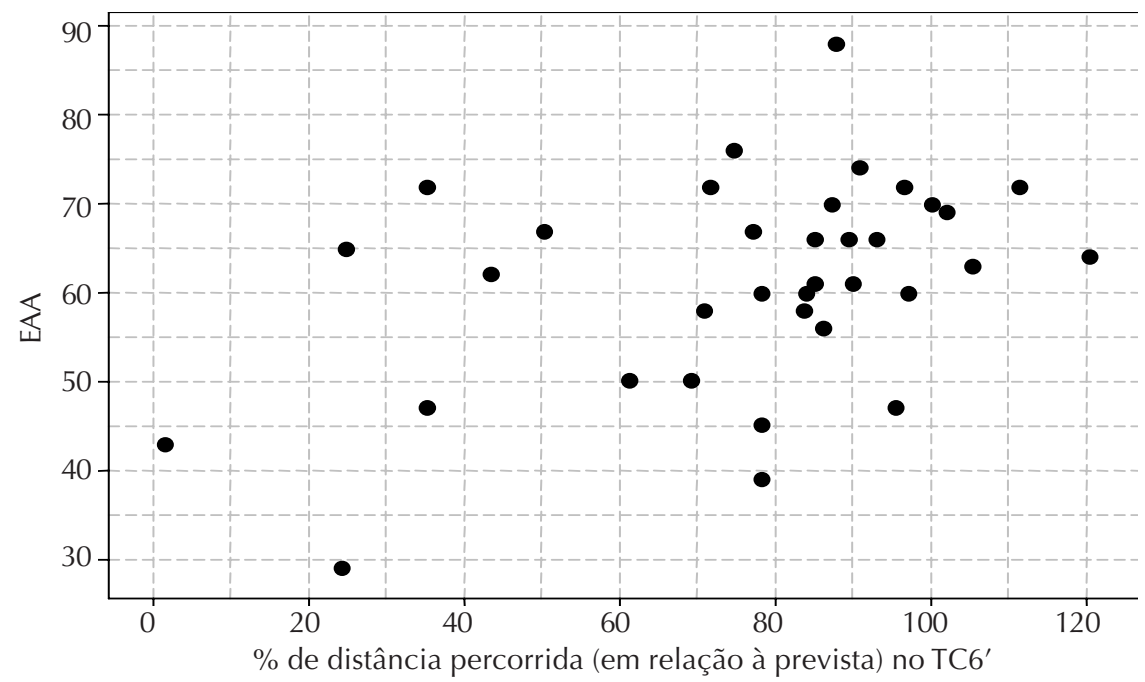

Gráfico 3 Correlação entre os escores ajustados de atividade (EAA) obtidos no $\mathrm{PAH}$ e as porcentagens da distância prevista percorrida no teste da caminhada de 6 minutos (TC6 ${ }^{\prime}$ )

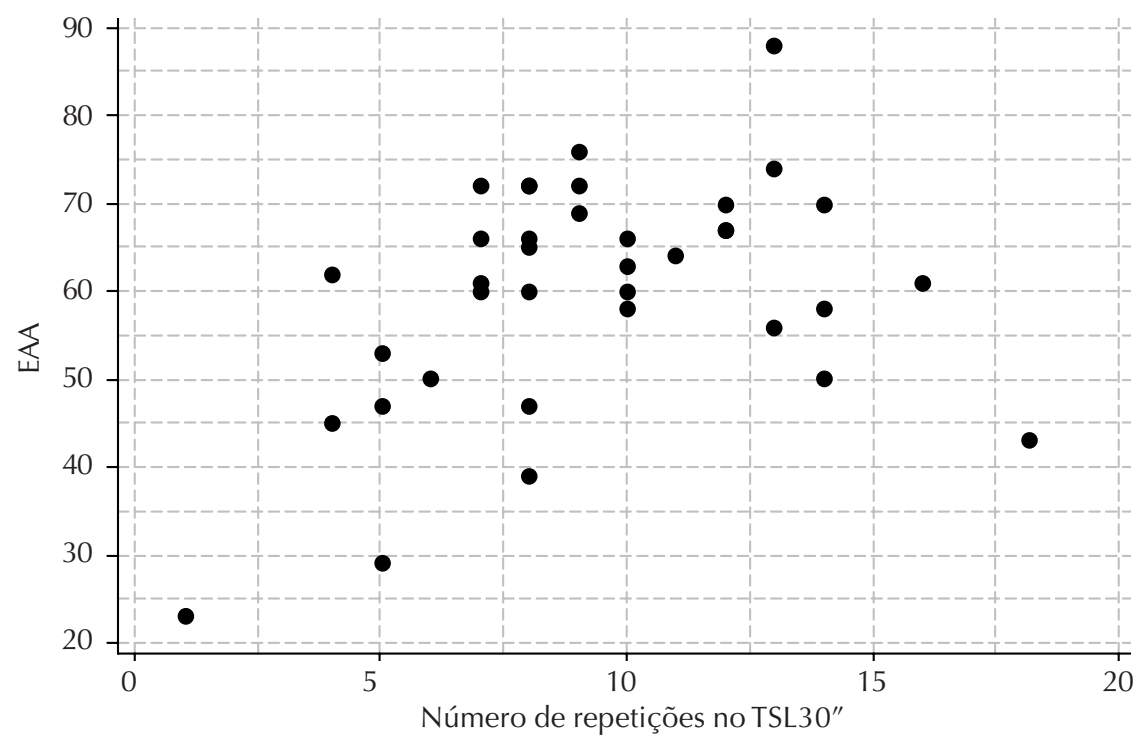

Gráfico 4 Correlação entre os escores ajustados de atividade (EAA) obtidos no PAH e o número de repetições no teste de levantar da cadeira em 30 segundos (TSL30")
$\mathrm{Na}$ análise da correlação entre o $\mathrm{PAH}$ e o teste de levantar da cadeira, também foi encontrada correlação positiva fraca $(r=0,36)$ e estatisticamente significante $(p=0,02)$ na amostra estudada (Gráfico 4).

Não houve correlação significante $(p=0,35)$ entre a classificação funcional obtida pelo PAH e o TSL30". As médias de repetições do teste de levantar da cadeira nos grupos moderadamente ativo e prejudicado foram 9,7 e 7,6 repetições, respectivamente. As três mulheres do grupo ativo foram excluídas desta análise em vista do número insuficiente para comparação.

\section{DISCUSSÃO}

No presente estudo encontrou-se maior concentração de idade na faixa de 70 a 79 anos (46\%) e idade média

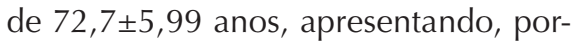
tanto, diferenças em relação aos idosos da comunidade de um estudo multicêntrico realizado em área metropolitana na região Sudeste do Brasil, cuja população analisada era relativamente mais jovem, com idade média de 69 anos e 58\% abaixo de 70 anos $^{29}$.

Segundo o auto-relato das pacientes, 78,3\% eram hipertensas, mas estudos epidemiológicos de vários países mostram prevalências de hipertensão arterial sistêmica (HAS) variando de $1 \%$ em regiões rurais de alguns países africanos até aproximadamente 30\%, entre operários urbanos da cidade de São Paulo ${ }^{30}$. Nossos achados indicam índices maiores, por se tratar de indivíduos idosos, pois a HAS tem na idade um forte fator de risco.

Apesar de 70,3\% da amostra não ter se considerado obesa ou com dificuldade em diminuir o peso corporal, foi encontrado IMC médio de 28 $\pm 4,7$ $\mathrm{kg} / \mathrm{m}^{2}$. De acordo com uma classificação do estado nutricional e do IMC adotado para o idoso, índices maiores de 27 indicam obesidade ${ }^{31}$, pois durante o processo de envelhecimento ocorre uma sensível perda de massa muscular e o correspondente ganho em massa de gordura, que aumenta em torno de 1,7\% em mulheres por década de vida, dos 15 aos 98 anos 
de idade ${ }^{32}$. Bennell et al. ${ }^{15}$ também encontraram valores semelhantes de IMC em pessoas com OA de joelhos $\left(29,3 \pm 4,2 \mathrm{Kg} / \mathrm{m}^{2}\right)$ e controles $(27,5 \pm 5 \mathrm{Kg} /$ $\mathrm{m}^{2}$ ), ambos grupos constituídos por idosos.

A média dos escores ajustados de atividade do PAH obtidas neste estudo foram inferiores às dos pacientes com artrite reumatóide e OA de Bilek et al. ${ }^{33}$ e às dos controles saudáveis de outros estudos ${ }^{15,17,18}$, tendo sido superiores às médias de pacientes em diálise ${ }^{18}$, acidente vascular encefálico ${ }^{17}$ e mulheres com OA de joelhos ${ }^{15}$.

$\mathrm{O}$ questionário $\mathrm{PAH}$ classificou a maior parte das idosas (70,2\%) como moderadamente ativa. Tal resultado seria esperado, já que geralmente existe um pequeno número de pessoas muito hábeis e outra pequena quantidade de pessoas muito debilitadas ${ }^{14}$. A própria amostra favorece esse resultado, por ter sido selecionada em ambulatório de atendimento de manutenção funcional para pacientes já reabilitados, integral ou parcialmente; os achados indicam justamente o público-alvo preferencial para tais ações em saúde. Supõe-se que idosos muito ativos tendem a procurar outras modalidades de assistência (centros de convivência, universidades abertas à terceira idade, academias) ou mesmo já incorporaram um estilo de vida ativo, mantendo-o por seus próprios meios, pela preservação de atividades domésticas, de trabalho e lazer.

Diversos estudos procuraram correlacionar o PAH a medidas de avaliação física como uma das formas de testar sua validade em populações distintas ${ }^{15-18,33,34}$. Com exceção de Bennell et al. ${ }^{15}$, que obtiveram correlações fracas a moderadas do PAH com medidas físicas (Timed up-andgo, step test e velocidade de marcha) e dor em pessoas com OA de joelhos, correlações fortes foram alcançadas com teste de consumo de oxigênio em esteira $^{33}$, tempo para levantar cinco ou dez vezes da cadeira ${ }^{18,33}$, caminhar 10 ou 15 metros $^{17,18,33}$, acelerômetro ${ }^{18}$ e tempo para subir escadas ${ }^{18}$. No estudo de Bennell et al. ${ }^{15}$, menos da metade da variância no $\mathrm{PAH}$ pôde ser explicada por uma combinação de testes de desempenho na análise de regressão múltipla, sugerindo que o PAH avalia uma dimensão diferente das outras medidas (de fato, níveis de atividade e dispêndio de energia), não fornecendo informação redundante quando em conjunto com outros instrumentos de avaliação em uma bateria de testes clínicos para reabilitação, sendo, portanto, complementar.

O TC6' não avalia diretamente o pico de consumo de oxigênio, embora essas medidas possam apresentar forte correlação ${ }^{19}$. Aplicado de modo a permitir que o sujeito empregue seu próprio passo durante o teste, a similaridade com as atividades de vida diária é supostamente maior, visto que essas atividades exigem níveis submáximos de esforço ${ }^{19}$. No presente estudo, as correlações entre as medidas no TC6', no TSL30" e o EAA do PAH estiveram presentes, porém, fracas. Sendo o EAA uma medida mais próxima das atividades cotidianas dos sujeitos, seriam esperadas correlações mais fortes entre as duas medidas.

O baixo grau de correlação encontrado indica uma distância entre o desempenho físico-funcional observado e a percepção que as pessoas têm de seu próprio nível de atividade. Assim como em qualquer questionário, é inerente ao PAH a influência da subjetividade nos resultados, podendo refletir-se em superestimação ou subestimação da capacidade real do sujeito $^{19,33}$. Outra limitação do PAH refere-se ao fato de que o sujeito é questionado sobre o que realmente já fez e parou de fazer, ou o que ainda faz, não contemplando o fazer com dificuldade, limitação ou dor - uma realidade presente no paciente em fase de reabilitação ${ }^{33}$. Pode-se ponderar que o nível de atividade física relatado é influenciado, portanto, por outras variáveis que não apenas o desempenho físico, tais como senso de controle, auto-eficácia, motivação, e dimensões contextuais como oportunidade, escolaridade, acesso a ambientes físicos atrativos, nível socioeconômico, além do sexo e hábitos como o tabagismo ${ }^{35}$.
Neste estudo, os valores de classificação de atividade não se correlacionaram com os índices alcançados no $\mathrm{TC}^{\prime}$, em parte, pelo pequeno número da amostra e por particularidades da amostra, selecionada a partir de sua inserção em serviço ambulatorial especializado. Outro aspecto relevante é que $6,5 \%$ dos itens do $\mathrm{PAH}$ em sua aplicação com idosos são erráticos: não se encaixando no contínuo de habilidade, prejudicam a mensuração da CF como um construto unidimensional ${ }^{14}$. É necessária cautela na aplicação desse instrumento, principalmente quando se tratar de população desfavorecida econômica e culturalmente, dada a variabilidade das respostas por dificuldade de compreensão ou por não desenvolvimento de uma determinada atividade física ao longo da vida por razões meramente contextuais.

Quando se compara a média das repetições do teste de levantar da cadeira em 30" entre os dois grupos de classificação funcional (moderadamente ativo e prejudicado), não são observadas diferenças estatisticamente significantes $(p=0,35)$. Em estudo realizado por Jones et al..$^{23} \mathrm{com}$ idosos da comunidade, foram encontradas médias de repetições para o TSL30" de 13,3 para o grupo mais ativo e 10,8 para o grupo menos ativo. Os presentes resultados mostram menores médias para os grupos moderadamente ativo e prejudicado (9,7 e 7,6 respectivamente), não sendo possível compará-las com a pesquisa dos autores supracitados, pois naquele estudo não há descrição do critério de classificação de atividade adotado. Quanto à média geral de repetições (9), foi mais baixa que a encontrada por Jones et al.23, que foi de 12,7 repetições em 42 muIheres idosas.

A maioria dos sujeitos do presente estudo $(65,7 \%)$ percorreu acima de $75 \%$ da distância prevista no TC6 segundo a equação de referência, sendo a mediana da amostra de $84 \%$. Mesmo em se tratando de amostra ambulatorial, é oportuno enfatizar termos encontrado resultados dentro da normalidade, em concordância com 
dados de pesquisas prévias: Enright et al. ${ }^{21}$ observaram que o $5^{\circ}$ percentil da distância do TC6' percorrida por participantes saudáveis - o qual pode ser considerado o limite mais baixo da faixa de normalidade - foi de $75 \%$ do valor predito.

Devido às limitações metodológicas do estudo, tais como tamanho reduzido da amostra e o fato de ter sido selecionada em um serviço de reabilitação física de um centro público, sugerem-se novas pesquisas com amos- tras maiores, representativas da população residente na comunidade, para que se possa inferir se a medida auto-referida está mais fortemente associada à medida observada ou se ocorre subestimação ou superestimação de desempenho quando a idosa é questionada sobre seu nível de atividade física.

\section{CONCLUSÃO}

Trata-se de um estudo de caráter preliminar cujos resultados permitem afir- mar que os níveis de classificação funcional e de atividade física, obtidos pelo Perfil de Atividade Humana, correlacionaram-se fracamente (nos casos em que as variáveis foram tomadas quantitativamente) ou apenas tenderam à correlação (nos casos de variáveis categóricas) com o desempenho físico avaliado por meio dos testes de caminhada de 6 minutos e de levantar da cadeira em 30 segundos, das idosas ambulatoriais que iniciariam um programa de reabilitação preventiva.

\section{REFERENCIAS}

1 IBGE - Instituto Brasileiro de Geografia e Estatística. Perfil dos idosos responsáveis pelos domicílios no Brasil, 2000. Rio de Janeiro; 2002.

2 Carvalho Filho ET. Fisiologia do envelhecimento. In: Papaléo-Netto M, Ponte JR, editores. Gerontologia: a velhice e o envelhecimento em visão globalizada. São Paulo: Atheneu; 2002. p.60-70.

3 Papaléo-Netto $M$, Ponte JR. Envelhecimento: desafio na transição do século. In: Papaléo-Netto $M$, Ponte JR, editores. Gerontologia: a velhice e o envelhecimento em visão globalizada. São Paulo: Atheneu 2002. p.3-12.

4 Kirkendall DT, Garrett Jr WE. The effects of aging and training on skeletal muscle. Am L Sports Med. 1998;26(4):598-602.

5 Hameed M, Harridge SD, Goldspink G. Sarcopenia and hypertrophy: a role for insulin-like growth factor1 in aged muscle? Exerc Sport Sci Rev. 2002;30(1):15-9.

6 Bemben MG, Massey BH, Bemben DA, Misner JE, Boileau RA. Isometric muscle force production as a function of age in healthy 20- to 74-yr-old men. Med Sci Sport Exerc. 1991;23(11):1302-10.

7 Daley MJ, Spinks WL. Exercise, mobility and aging. Sports Med. 2000;29(1):1-12.

8 Pires CMR. Fisiologia do exercício aplicada ao idoso. In: Rebelatto JR, Morelli JGS, editores. Fisioterapia geriátrica: a prática da assistência ao idoso. São Paulo: Manole; 2004. p.123-65.

9 Posner JD, McCully KK, Landsberg LA, Sands LP, Tycenski P, Hofmann MT, et al. Physical determinants of independence in mature women. Arch Phys Med Rehabil. 1995;76(4):373-80.
10 Higginbotham MB, Morris KG, Williams RS, Coleman RE, Cobb FR. Physiologic basis for the agerelated decline in aerobic work capacity. Am J Cardiol. 1986;57(15):1374-9

11 Cordeiro RC. Reabilitação gerontológica. In: Ramos LR, Toniolo-Neto J, editores. Guias de medicina ambulatorial e hospitalar Unifesp - Escola Paulista de Medicina: Geriatria e Gerontologia. São Paulo: Manole; 2005. p.209-27.

12 Ramos LR. A mudança de paradigma na saúde e o conceito de capacidade funcional. In: Ramos LR, TonioloNeto J, edirores. Guias de medicina ambulatorial e hospitalar Unifesp - Escola Paulista de Medicina: Geriatria e Gerontologia. São Paulo: Manole; 2005. p.1-7.

13 Ramos LR, Simoes EJ, Albert MS. Dependence in activities of daily living and cognitive impairment strongly predicted mortality in older urban residents in Brazil: a 2year follow-up. J Am Geriatr Soc. 2001;49(9):1168-75.

14 Souza AC, Magalhaes LC, Teixeira-Salmela LF. Cross-cultural adaptation and analysis of the psychometric properties in the Brazilian version of the Human Activity Profile. Cad Saude Publica. 2006;22(12):2623-36.

15 Bennell KL, Hinman RS, Crossley KM, Metcalf BR, Buchbinder R, Green S, et al. Is the Human Activity Profile a useful measure in people with knee osteoarthritis? J Rehabil Res Dev. 2004;41(4):621-30.

16 Farrell MJ, Gibson SJ, Helme RD. Measuring the activity of older people with chronic pain. Clin J Pain. 1996;12(1):6-12.

17 Teixeira-Salmela LF, Devaraj R, Olney SJ. Validation of the Human Activity Profile in stroke: a comparison of observed, proxy and self-reported scores. Disabil Rehabil. 2007;29(19):1518-24. 


\section{Referências (cont.)}

18 Johansen KL, Painter P, Kent-Braun JA, Ng AV, Carey $S$, Da Silva $M$, et al. Validation of questionnaires to estimate physical activity and functioning in endstage renal disease. Kidney Int. 2001;59(3):1121-7.

19 ATS statement: guidelines for the six-minute walk test. Am J Respir Crit Care Med. 2002;1;166(1):111-7.

20 McArdle WD, Katch FI, Katch VL. Transferência de energia no exercício. In: McArdle WD, Katch FI, Katch VL, editores. Fisiologia do exercício: energia, nutrição e desempenho humano. Rio de Janeiro: Guanabara Koogan; 2003. p.161-78.

21 Enright PL, McBurnie MA, Bittner V, Tracy RP, McNamara R, Arnold A, et al. The 6-min walk test: a quick measure of functional status in elderly adults. Chest. 2003;123(2):387-98.

22 Enright PL, Sherrill DL. Reference equations for the six-minute walk in healthy adults. Am J Respir Crit Care Med. 1998;158(5Pt 1):1384-7.

23 Jones CJ, Rikli RE, Beam WC. A 30-s chair-stand test as a measure of lower body strength in communityresiding older adults. Res Q Exerc Sport. 1999;70(2):113-9.

24 McArdle WD, Katch FI, Katch VL. Diferenças individuais e mensuração das capacidades energéticas. In: McArdle WD, Katch FI, Katch VL, editores. Fisiologia do exercício: energia, nutrição e desempenho humano. Rio de Janeiro: Guanabara Koogan; 2003. p.228-9.

25 Massey Jr FJ. The Kolmogorov-Smirnov test of goodness of fit. J Am Statist Assoc. 1951;46:70-4.

26 Snedecor GW, Cochran WG. The sample correlation coefficient "r" and "properties of $r " \S 10.1-10.2$. Statistical methods. Ames: lowa State Press; 1980. p.175-8.
27 Bland M. An Introduction to medical statistics. Oxford: Oxford University Press; 2001.

28 Walker G. Common statistical methods for clinical research with SAS examples. Cary: SAS Institute; 2002.

29 Ramos LR, Rosa TE, Oliveira ZM, Medina MCG, Santos FRG. Perfil do idoso em área metropolitana na região sudeste do Brasil: resultados de inquérito domiciliar. Rev Saude Publica. 1993;27(2):87-94.

30 Olmos RD, Lotufo PA. Epidemiologia da hipertensão arterial no Brasil e no mundo. Rev Bras Hipertens. 2002;9(1):21-3.

31 Najas M, Nebuloni CC. Avaliação do estado nutricional. In: Ramos LR, Toniolo-Neto J, editores. Guias de medicina ambulatorial e hospitalar Unifesp - Escola Paulista de Medicina: Geriatria e Gerontologia. São Paulo: Manole; 2005. p.299-314.

32 Soares J, Alabarse SL. Envelhecimento e atividade física. In: Ramos LR, Toniolo-Neto J, eds. Guias de medicina ambulatorial e hospitalar Unifesp - Escola Paulista de Medicina: Geriatria e Gerontologia. São Paulo: Manole; 2005. p.255-70.

33 Bilek LD, Venema DM, Camp KL, Lyden ER, Meza JL. Evaluation of the human activity profile for use with persons with arthritis. Arthritis Rheum. 2005;53(5):756-63.

34 Davidson M, Morton N. A systematic review of the Human Activity Profile. Clin Rehabil. 2007;21(2):151-62.

35 Zaitune MP, Barros MB, Cesar CL, Carandina L, Goldbaum M. Variables associated with sedentary leisure time in the elderly in Campinas, Sao Paulo State, Brazil. Cad Saude Publica. 2007;23(6):1329-38. 